\title{
Mortalidad Materna en el Instituto Materno Infantil de Bogotá Años: 1981 - 1982
}

\author{
Dr. Epifanio Becerra Corredor
}

\section{INTRODUCCION}

La tasa de mortalidad materna es un fiel indicador de la calidad de los programas que en atención a la embarazada brinda un país o un organismo de salud determinado. Es posible deducir a través de esta tasa el desarrollo cultural, social, económico y tecnológico alcanzado en el lugar donde se analizan sus resultados, $(6,14)$.

Acorde con las consideraciones anteriores es fácil entender por qué en países desarrollados donde se dan las condiciones necesarias para garantizar una atención integral de la mujer grávida, las tasas de mortalidad no sobrepasan en un 5/ 10.000 nacidos vivos, con cifras mínimas de $0.4 / 10.000$ como las encontradas en Dinamarca, $(2,5,6,13)$. En cambio en los países del tercer mundo, esta cifra se eleva en forma dramática y es así como encontramos tasas que van desde un 10 a un 35/10.000 nacidos vivos, $(1,2$, $4,12,17)$.

En nuestro país a pesar de existir un subregistro estadístico importante se tiene de acuerdo con los trabajos adelan- tados sobre mortalidad materna en los últimos años en diferentes Hospitales Universitarios, y más recientemente en el estudio cooperativo coordinado por el Centro Regional de Población, cifras comprendidas entre 20 y $33.7 / 10.000$ nacidos vivos $(3,7,8,19)$.

\section{Mortalidad Materna en el I.M.I.}

El Instituto Materno Infantil de Bogotá (|MI) es un Hospital especializado destinado a la atención de pacientes de alto riesgo obstétrico referida de los diferentes organismos de salud que conforman la Regional No. 3. En la práctica a esta Institución llegan pacientes de recursos económicos y de nivel socio-cultural bajo, provenientes no sólo de la ciudad de Bogotá, sino de otras poblaciones y Departamentos vecinos. Al no existir una verdadera regionalización ni unificación en las tarifas, entre los diferentes Hospitales encargados de la atención obstétrica, la Institución en mención tiene una demanda de servicios que sobrepasa en mucho sus recursos físicos y presupuestales. 
Al revisar los estudios sobre mortalidad materna realizados en los últimos veinte (20) años en el Instituto Materno Infantil (IMI), y usando el mismo denominador (10.000 nacidos vivos)len todo ellos, se encuentra las siguientes tasas: $(8,10,15,16,17)$.

\begin{tabular}{lll}
\hline Autor & Años & Tasa \\
\hline López Ruíz & $1960-65$ & 45.4 \\
Rojas y Cols. & $1966-75$ & 35 \\
Sánchez T. & $1970-75$ & 30.47 \\
Muñoz y Cols. & $1976-80$ & 25.04 \\
\hline
\end{tabular}

Los resultados en los diferentes períodos analizados muestran un descenso de la mortalidad materna en el Instituto Materno Infantil (IMI). Al compararla con las cifras encontradas en otras Instituciones similares de Latinoamérica, se observa que ésta permanece aún muy elevada $(1,4,12)$.

El presente trabajo tiene entre sus objetivos; establecer la tasa de mortalidad materna en el Instituto Niaterno Infantil (IMI) durante los años 1981-1982, lapso que coincide con el cierre del Hospital y traslado provisional de los diferentes servicios a un área reducida del Hospital San Juan de Dios, mientras se remodela su planta física.

El cambio de sede, trajo entre otras las siguientes consecuencias:

1. Reducción de la capacidad instalada del Instituto en sus áreas de hospitalización a un $10 \%$.

2. Mayor congestión y hacinamiento en áreas de internación de pacientes.

3. Aumento en circulación del personal de estudiantes, médicos y para-médicos en áreas restringidas.
4. Uso de quirófanos cercanos a salas destinadas a procedimientos sépticos.

5. Utilización de la sala de recuperación post-quirúrgica general, en donde por falta de camas en el Hospital se internan pacientes con diferentes patologías y se permite la presencia de visitantes.

\section{OBJETIVOS}

1. Determinar la tasa de mortalidad materna en el Instituto Materno Infantil (IMI) durante los años 1981-1982.

2. Conocer las causas más frecuentes de mortalidad materna en el Instituto Materno Infantil (IMI) y analizar las variables determinantes.

3. Establecer una tasa depurada de mortalidad materna propia del Instituto Materno Infantil (IMI).

\section{TIPO DE ESTUDIO - UNIVERSO}

Se hace un trabajo de tipo descriptivorectrospectivo. La población a estudiar está dada por las pacientes obstétricas faIlecidas en el Instituto Materno Infantil (IMI) durante los años 1981-1982.

\section{RECOLECCION DE LA INFORMACION- TABULACION}

La información requerida se obtuvo directamente de las historias clínicas en el Departamento de Estadística y del archivo de Patología del Instituto. En la recolección de los datos se utilizó un formulario que contiene las variables establecidas previamente en el protocolo de la presente investigación. El formulario consta de 3 partes a saber:

A - Identificación de la paciente.

B - Contenido de variables a estudiar. 
C - Comentarios donde el encuestador hace las anotaciones o aclaraciones pertinentes.

\section{DEFINICION DE VARIABLES}

\section{Muerte materna}

"Es el fallecimiento de cualquier mujer por cualquier causa mientras está embarazada o dentro de cierto período después de la terminación del embarazo independiente de la duración o del sitio del mismo".

Este período fue aprobado por la FIGO de 42 días.

La muerte materna se clasifica en tres grupos:

a-Muerte materna directa:

(Anteriormente mortalidad obstétrica).

"Es el fallecimiento resultante de complicaciones obstétricas del embarazo, trabajo de parto y puerperio; o intervenciones, omisiones de tratamiento necesario, tratamien to incorrecto o combinación de estas causas".

$b$ - Muerte materna indirecta:

(Anteriormente muerte obstétrica indirecta).

“Es el fallecimiento obstétrico resultante de enfermedad previa o enfermedad desarrollada durante el embarazo, parto o puerperio que no es debida directamente a causas obstétricas sino que es agravada por los efectos funcionales del embarazo".

\section{c-Muerte no materna:}

(Anteriormente muerte no obstétrica);

"Fallecimiento obstétrico resultante de causas accidentales o circunstanciales, no relacionadas con el embarazo o su tratamiento"'.

\section{Tasa de muerte materna}

Es el número de muertes maternas directas, indirectas y no maternas por 10.000 nacidos vivos, en un período determinado.

\section{Nacidos vivos}

Se tuvo en cuenta la clasificación de la FIGO (1970). "Es la expulsión o extracción completa del producto de la concepción que pese más de 1.000 gramos o tenga más de 28 semanas de gestación; o en su defecto mida más de $35 \mathrm{cms}$., y que muestre señales de vida después de la separación de la madre".

\section{Edad}

Años cumplidos de la paciente según registro de la historia cl ínica.

\section{Procedencia}

Lugar en donde vivió la paciente en los últimos meses antes de su ingreso al hospital.

\section{Remisión}

Organismo de salud o persona que envió a la paciente al I.M.I.

\section{Gestaciones}

Número de embarazos anotados en la historia clínica.

\section{Edad gestacional}

Tiempo en semanas del embarazo al ingreso de la paciente, en relación con la fecha de la última menstruación.

\section{Riesgo obstétrico}

Se refiere a la probabilidad de presentar complicaciones durante el embarazo, parto o puerperio. 


$$
a-\text { Bajo riesgo: }
$$

Se clasifica así el embarazo en el cual la madre, el feto o el recién nacido tiene pocas posibilidades de presentar complicaciones.

\section{$b$ - Alto riesgo:}

Se denomina embarazo de alto riesgo aquel en donde la madre, el feto o el recién nacido están o estarán expuestos a mayores peligros, en relación con un embarazo normal.

\section{Causa de muerte}

Corresponde a la registrada en la Hoja de Egreso con base en el diagnóstico clínico $\mathrm{y} / \mathrm{o}$ estudio de anatomía patológica.

\section{Muerte Institucional}

Es la muerte materna ocurrida en el I.M.I. después de 24 horas de hospitalización.

\section{Muerte no Institucional}

Toda muerte materna ocurrida en e! I.M.I. antes de 24 horas de hospitalización, siempre y cuando la paciente haya ingresado con una complicación debida o no a su embarazo.

\section{Atención médica}

Se refiere a la calidad del manejo dispensado a cada paciente en cuanto al diagnóstico, tratamiento y/o seguimiento por parte del personal médico de la Institución. Tiene las siguientes subcategorías:

\section{$a$ - Atención médica buena:}

Cuando el diagnóstico, tratamiento y/o seguimiento en cada paciente cumple con las normas establecidas en el
Departamento de Gineco-Obstetricia del I.M.I.

\section{$b$ - Atención médica regular:}

Cuando existe error $u$ omisión en el diagnóstico, tratamiento $\mathrm{y} / \mathrm{o}$ seguimiento de la paciente sin que esto incidiera directamente en el resultado final.

\section{$c$ - Atención médica mala:}

Siempre que se compruebe error $u$ omisión en el diagnóstico, tratamiento y/o seguimiento de la paciente $v$ esto influya en forma desfavorable en la evolución del estado clínico.

\section{Atención Institucional}

Se refiere a la disponibilidad de los recursos humanos y físicos necesarios en el diagnóstico, tratamiento $\mathrm{y} / \mathrm{o}$ seguimiento de las pacientes que ingresan al I.M.I.

Se consideran tres (3) sub-categorías:

$$
a-\text { Atención Institucional buena: }
$$

Es cuando el hospital cuenta con personal médico y paramédico suficiente; así como disponibilidad de elementos necesarios para un adecuado diagnóstico, tratamiento $\mathrm{y} / \mathrm{o}$ seguimiento de las pacientes que ingresan al servicio.

\section{$b$ - Atención Institucional regular:}

Se califica así la atención institucional, cuando no existen todos los recursos humanos y físicos considerados necesarios en el manejo integral de la paciente.

\section{c-Atención Institucional mala:}

Cuando no se cuenta con los medios necesarios para el diagnóstico, tratamiento $\mathrm{y} / \mathrm{o}$ seguimiento de la paciente $\mathrm{y}$ 
esta situación incide negativamente en la evolución posterior.

\section{RESULTADOS}

\section{Tasa de mortalidad}

En los dos (2) años estudiados (19811982) se registraron en el I.M.I. sesenta y ocho (68) muertes de pacientes obstétricas de las cuales se descartan dos (2) por sobrepasar el límite de los 42 días establecidos por la FIGO. Durante este mismo lapso ocurrieron 20.524 nacimientos de niños vivos.

De esta manera tenemos en el período revisado una tasa de mortalidad materna promediada de $32.64 \times 10.000$ nacidos vivos. Los datos anteriores se muestran por año en los cuadros 1, 2 y 3 respectivamente.

Tabla No. 1

\begin{tabular}{lrr}
\hline Año & 1981 & 1982 \\
Muertes & 36 & 30 \\
\hline
\end{tabular}

Tabla No. 2

NAVIDOS VIVOS

\begin{tabular}{llll}
\hline Año & $\mathbf{1 9 8 i}$ & $\mathbf{1 9 8 2}$ & Total \\
& 9.381 & 11.143 & 20.524 \\
\hline
\end{tabular}

Tabla No. 3

\section{TASA POR AÑO 1981}

$\frac{36 \times 10.000}{9.381}=38.37 \times 10.000$ Nacidos

\section{2}

$\frac{30 \times 10.000}{11.143}=26.96 \times 10.000 \underset{\text { vivos }}{\text { Nacidos }}$

\section{Causas de mortalidad materna}

Al agrupar las muertes de acuerdo con la clasificación de la FIGO se encontró 58 de ellas de causa directa $(87.8 \%) ; 6$ de causa indirecta $(9.1 \%)$; y dos de causa no relacionada (3.1\%). Ver tabla 4.

Tabla No. 4

\section{CLASIFICACION DE LA MORTALIDAD}

\begin{tabular}{lrrrc}
\hline Año & 1981 & 1982 & Total & Porcentaje \\
\hline Directa & 31 & 27 & 58 & 87.8 \\
Indirecta & 5 & 1 & 6 & 9.1 \\
No obstétrica & - & 2 & 2 & 3.1 \\
\hline Total & 36 & 30 & 66 & 100 \\
\hline
\end{tabular}

\section{A - MUERTE MATERNA DIRECTA}

La mayor mortalidad se debió a complicaciones obstétricas ocurridas en los diferentes períodos de la gestación y el puerperio (58 casos). Los factores desencadenantes del deceso se relacionan en la Tabla 5.

Tabla No. 5

MUERTE DIRECTA

\begin{tabular}{lrrrc}
\hline Año & 1981 & 1982 & Total & Porcentaje \\
\hline Infección & 27 & 23 & 50 & 86.2 \\
Toxemia & 2 & 2 & 4 & 6.9 \\
Hemorragia & 2 & 1 & 3 & 5.2 \\
$\begin{array}{l}\text { Embolia de líquido } \\
\text { amniótico }\end{array}$ & 31 & 1 & 1 & 1.7 \\
\cline { 2 - 5 } & & 27 & 58 & 100.0 \\
\hline
\end{tabular}


La infección como consecuencia del aborto, parto o cesárea ocupa el primer lugar de las causas directas (86.2\%), seguida de la Toxemia (6.9\%) y la $\mathrm{He}$ morragia (5.2\%).

\section{Mortalidad materna por infección}

Del total de muertes maternas ocurridas en estos dos (2) años estudiados 50 de ellas (75.7\%) tuvieron en la infección un común denominador. La Tabla 6 muestra el estudio gestacional donde ésta se produjo.

Tabla No. 6

INFECCION

\begin{tabular}{lrrrc}
\hline Año & $\mathbf{1 9 8 1}$ & $\mathbf{1 9 8 2}$ & Total & Porcentaje \\
\hline Post-aborto & 16 & 18 & 34 & 68 \\
Post-ectópico & - & 1 & 1 & 2 \\
Post-parto & 3 & 4 & 7 & 14 \\
Post-cesárea & 8 & 0 & 8 & 16 \\
\hline & 27 & 23 & 50 & 100 \\
\hline
\end{tabular}

La sepsis ocurrió con mayor incidencia después de abortos provocados (68\%).

Llama la atención el elevado porcentaje de infección encontrado después de parto o cesárea. Al revisar estos casos se observó lo siguiente: Del total de los (7) partos complicados, cuatro (4) de ellos fueron atendidos en otras Instituciones y remitidos al I.M.I. en estado de sepsis avanzada. Los tres restantes fueron atendidos en el Hospital, dos se complicaron después del parto normal y uno presentaba al ingreso severa amnionitis.

Hubo ocho (8) pacientes cuya sepsis se presentó después de una cesárea; cinco (5) de ellas operadas en hospital regional, ingresaron al I.M.I. en estado de peritonitis.
Tres pacientes intervenidas en el Hospital presentaron dehiscencia de la Histerorrafia y Peritonitis.

Tabla No. 7

TOXEMIA

\begin{tabular}{ccc}
\hline Año & 1981 & 1982 \\
Casos & 2 & 2 \\
\hline
\end{tabular}

Dentro de este grupo se consideró solamente aquellas pacientes a quienes se comprobó plenamente el diagnóstico de toxemia pura. Se excluyeron los casos con antecedentes de enfermedad vascular crónica hipertensiva (E.V.C.H.).

Las cuatro pacientes en quienes se demostró claramente la existencia de toxemia pura, llegaron al I.M.I. después de haber presentado cuadro de eclampsia. A su ingreso se diagnósticó embarazo a término y accidente cerebro vascular (A.C.V.) de tipo hemorrágico; se les instauró tratamiento médico adecuado y se procedió a desembarazarlas; dos de ellas por reforzamiento del trabajo de parto y dos por intervención cesárea. A consecuencia de la complicación neurológica murieron dentro de un término no mayor de 48 horas.

Tabla No. 8

HEMORRAGIA

\begin{tabular}{lc}
\hline Causa & Número de Casos \\
\hline Placenta previa & 1 \\
Abruptio placenta & 1 \\
Coagulación intravascular & 1 \\
diseminada (C.I.D.) & \\
\hline
\end{tabular}


La hemorragia como causa de muerte ocupa el tercer lugar de las causas directas y el cuarto lugar dentro de la mortalidad global. Los factores determinantes de ella se presentan en la Tabla 8. Vale la pena aclarar que el caso de (C.I.D.), se presentó después de practicar una histerectomía total abdominal en bloque a una mujer a quien se diagnósticó enfermedad trofoblástica.

El caso de muerte por placenta previa ocurrió en una mujer que ingresó al Hospital en estado de shock severo por hemorragia y a quien se le practicó intervención cesárea de inmediato; previa aplicación de líquidos. No se administró sangre por no encontrarse la correspondiente a su grupo sanguíneo $y$ fallece durante la intervención.

El caso de abruptio se diagnosticó oportunamente $y$ después de operación cesárea en el postoperatorio inmediato aparece cuadro de hemorragia uterina severa y se practica histerectomía total. Fallece pocas horas después de salir de cirugía a consecuencia de C.I.D.

\section{B - MORTALIDAD MATERNA INDIRECTA}

Fue fácil demostrar la existencia de una patología previa al embarazo, agravada por el estado grávido puerperal en 6 casos $(9.09 \%$ ) los cuales se relacionan en la Tabla 9 .

Tabla No. 9

\section{MUERTE INDIRECTA}

\begin{tabular}{lcccc}
\hline & 1981 & 1982 & Total & Porcentaje \\
\hline $\begin{array}{l}\text { Enfermedad vascular } \\
\text { crónica hipertensiva }\end{array}$ & 4 & - & 4 & 66.6 \\
Cáncer del seno & 1 & - & 1 & 16.7 \\
Diabetes & - & 1 & 1 & 16.7 \\
\hline
\end{tabular}

Las cuatro pacientes muertas por enfermedad vascular crónica hipertensiva concomitante con el embarazo fallecieron a consecuencia de accidentes cerebrovasculares tipo hemorrágico.

Una paciente con carcinoma de seno estado IV ingresó al I.M.I. con un embarazo de 30 semanas. La conducta inmediata consistió en inducción del parto el cual se presentó en las siguientes 24 horas de su admisión; desafortunadamente falleció al décimo quinto día de puerperio en un estado de carcinomatosis generalizada.

Una paciente diabética con embarazo de 32 semanas y tratamiento médico adecuado presentó parto con feto muerto a las 12 horas de su ingreso $y$ fallece 2 días después de la hospitalización.

\section{C - MUERTE NO MATERNA}

Tabla No. 10

MUERTE NO MATERNA

\begin{tabular}{lcc}
\hline Diagnostico & Casos & Año \\
\hline Hepatitis & 1 & 1982 \\
Apendicitis perforada & 1 & 1982 \\
\hline
\end{tabular}

Hubo dos casos de muertes no materna cuya causa no estaba relacionada con el embarazo. Uno de ellos fue remitido de un Hospital Comunitario al segundo día de puerperio, con un cuadro caracterizado por ictericia a consecuencia de una hepatitis severa; fallece antes de 24 horas de su internación.

El segundo caso se trataba de una paciente puerpera de 20 días que ingresa al Instituto con una peritonitis generalizada a partir de una apendicitis perforada; no responde al tratamiento médicoquirúrgico y fallece en sepsis generalizada. 


\section{Mortalidad Materna en relación con Edad y Causa}

La mortalidad materna según edad y causa se relaciona en la Tabla 11.

En el grupo de mujeres de 20-29 años (59.1은) se presentó una mayor mortalidad; su causa fue directa a expensas de la infección.

Sigue en frecuencia el grupo de mujeres mayores de 35 años $(21.2 \%$ ) en donde existe sensiblemente igual número de causas directa e indirecta determinada por la infección, la hemorragia y la enfermedad vascular crónica hipertensiva.
En el grupo de mujeres de $20-29$ años (59.1을 se presentó una mayor mortalidad; su causa fue directa a expensas de la infección.

Sigue en frecuencia el grupo de mujeres mayores de 35 años (21.2\%) en donde existe sensiblemente igual número de causas directa e indirecta determinadas por la infección, la hemorragia y la enfermedad vascular crónica hipertensiva.

Distribución de la mortalidad materna según gestaciones y causa. Se analizó la mortalidad materna según el número de gestaciones y causa de muerte lo cual se relaciona en la Tabla 12.

Tabla No. 11

EDAD Y CAUSA

\begin{tabular}{lccccccc}
\hline Edad & Infección & Toxemia & Hemorragia & Otras & Indirecta & No orstétrica & Porcentaje \\
\hline $15-19$ & 5 & 3 & - & - & - & - & 12.1 \\
$20-24$ & 17 & - & - & - & - & 1 & 27.3 \\
$25-29$ & 19 & 1 & - & 1 & - & - & 31.8 \\
$30-34$ & 4 & - & - & - & 1 & - & 7.6 \\
35 y más & 5 & - & 3 & - & 5 & 1 & 21.2 \\
\hline
\end{tabular}

Tabla No. 12

NUMIERO DE GESTACIONES Y CAUSA

\begin{tabular}{crrrrrrr}
\hline Gestaciones & Infección & \multicolumn{1}{c}{ Toxemia } & Hemorragia & Otras & Indirecta No obstétrica & Total \\
\hline G1 & 10 & 2 & - & - & - & 1 & 13 \\
G2 & 4 & - & - & 1 & 2 & - & 7 \\
G3 & 10 & 1 & - & - & 1 & - & 12 \\
G4 & 9 & 1 & - & - & - & - & 10 \\
G5 y más & 17 & - & 3 & - & 3 & 1 & 24 \\
\hline
\end{tabular}


Se encontró una mayor mortalidad en el grupo de mujeres con más de 4 gestaciones ( 34 casos), o sea un 51.5\%. La causa estuvo determinada especialmente por la infección.

Al grupo anterior sigue las pacientes que tan sólo cursaban su primer embarazo (19.6\%); como el anterior tuvieron en la infección su causa de muerte.

Tabla No. 13

\section{LUGAR DE REMISION}

\begin{tabular}{lrc} 
Lugar & Número & Porcentaje \\
\hline Hospital universitario & 3 & 9.1 \\
Hospital regional & 15 & 45.5 \\
Hospital comunitario & 9 & 27.2 \\
Centro de Salud & 6 & 18.2 \\
\hline
\end{tabular}

Consultaron al I.M.I. directamente 33 pacientes $50 \%$.

Llama la atención en la Tabla anterior como el $50 \%$ de las pacientes fallecidas consultaron primero a otras Instituciones. De este grupo 24 mujeres (72.7\%) fueron atendidas inicialmente en hospitales de atención intermedia de Bogotá, después de parto, cesárea o raspado presentaron cuadro de sepsis que obligó su traslado al I.M.I.

En otros casos (9.1\%) las pacientes consultaron a hospitales universitarios, en donde a pesar de existir los recursos necesarios para su atención, no se les prestó el servicio.

El $18.2 \%$ de las pacientes acudieron a organismos de atención primaria, donde por no contar con los medios adecuados se remitieron al nivel terciario.

El 50\% de las muertes maternas ocurrió cuando la paciente cursaba el primer trimestre gestacional a consecuencia del aborto provocado; salvo en un caso en donde la infección sobrevino en el postoperatorio de un embarazo extrauterino.

En un 33.3 (22 casos) la complicación determinante de la muerte se presentó en el puerperio. Predominó en este período la infección (14 casos) seguida de lejos por la toxemia (2 casos), la hemorragia ( 2 casos), la enfermedad vascular hipertensiva $y$ causas no obstétricas.

Durante el tercer trimestre las causas indirectas, especialmente la enfermedad vascular crónica hipertensiva complicada con A.C.V. hemorrágico determinó el fallecimiento de 5 pacientes (7.5\%).

De acuerdo con los parámetros de clasificación del riesgo obstétrico existentes en el Hospital, las pacientes fallecidas se clasificaron al ingreso como de alto riesgo en el $95.5 \%$ y de bajo riesgo en el $4.5 \%$.

Tabla No. 14

\section{PERIODO GESTACIONAL Y COMPLICACION}

\begin{tabular}{|c|c|c|c|c|c|c|c|}
\hline Estadio & Infección & Toxemia & Hemorragia & Otras & Indirecta & No obstétrica & Total \\
\hline 1er. trimestre & 33 & - & - & - & - & - & 33 \\
\hline 2o. trimestre & 2 & - & 1 & - & - & - & 3 \\
\hline 3er. trimestre & 1 & 2 & - & - & 5 & - & 8 \\
\hline Puerperio & 14 & 2 & 1 & 1 & 2 & 2 & 22 \\
\hline
\end{tabular}


Tabla No. 15

RIESGO OBSTETRICO

\begin{tabular}{lrr}
\hline Clasificación & Casos & Porcentaje \\
\hline Bajo riesgo & 3 & 4.5 \\
Alto riesgo & 63 & 95.5 \\
\hline
\end{tabular}

\section{Anatomía Patológica}

El examen de Anatomía Patológica se hizo a partir de muestras obtenidas después de intervenciones quirúrgicas y/o práctica de autopsia en algunas de las pacientes muertes. En la Tabla 16 se relacionan los resultados.

Tabla No. 16

\section{ESTUDIO ANATOMIA PATOLOGICA}

\begin{tabular}{lrc}
\hline & Casos & Porcentaje \\
\hline Piezas quirúrgicas solamente & 37 & 56 \\
Autopsia & 10 & 15 \\
Pieza quirúrgica más autopsia & 8 & 12 \\
Sin estudio & 11 & 16 \\
\hline
\end{tabular}

Los resultados anteriores muestran como en el $83 \%$ de las pacientes fallecidas fue posible confirmar el diagnóstico clínico con base en uno o dos estudios histopatológicos.

En 11 casos (16\%) no se practicó autopsia; de ellos 6 por existir demanda penal en donde se hizo necesario el levantamiento médico legal.

En los 5 restantes, los familiares se opusieron a la práctica de la autopsia.

En el 30:1\% de las muertes maternas se consideró que existió algún grado de falla en la disponibilidad de recursos
Tabla No. 17

\section{ATENCION INSTITUCIONAL}

\begin{tabular}{lrr}
\hline & Casos & Porcentaje \\
\hline Buena & 46 & 69.7 \\
Regular & 13 & 19.6 \\
Mala & 7 & 10.7 \\
\hline
\end{tabular}

necesarios por parte del Hospital para la adecuada atención. Sólo en un 10.7\% la falla institucional influyó desfavorablemente en la evolución de las pacientes.

Tabla No. 18

ATENCION MEDICA

\begin{tabular}{lcc}
\hline & Casos & Porcentaje \\
\hline Buena & 45 & 68.2 \\
Regular & 12 & 18.2 \\
Mala & 9 & 13.6 \\
\hline
\end{tabular}

En el $32 \%$ de las muertes maternas existió algún error en el diagnóstico de ingreso, en la evolución y/o manejo de la paciente. El error médico incidió desfavorablemente en la recuperación de las enfermas en un $13.6 \%$.

Tabla Nóo. 19

MUERTE INSTITUCIONAL

\begin{tabular}{lrc}
\hline & Casos & Porcentaje \\
\hline Menos de 24 horas & 10 & 15.2 \\
Más de 24 horas & 56 & 84.8 \\
\hline
\end{tabular}

De las 66 pacientes fallecidas en el I.M.I. durante los dos años analizados se aprecia que aproximadamente la sexta parte de las muertes se produjeron antes 
de 24 horas de su estadía en la Institución.

\section{DISCUSION}

El análisis de la mortalidad materna ocurrida en el I.M.I. durante los años 1981-1982 está basado en la metodología seguida en el estudio realizado sobre este mismo aspecto en el lapso comprendido entre 1976-1980.

Hemos querido con el presente trabajo continuar la revisión sobre este campo con el ánimo de mantener actualizada la situación sobre la atención materna dispensada en el nivel universitario de la Regional No. 3 de Bogotá; en donde necesariamente se refleja a través de este indicador la realidad que se vive en otras áreas de Bogotá y del país, que registran situaciones similares en razón al nivel de pacientes que se atienden y los recursos disponibles en la solución de los problemas de salud.

Los comentarios hechos en el trabajo mencionado anteriormente sobre la calidad del dato obtenido en la Historia Clínica, son válidos en el presente estudio. Entre las fallas detectadas con más frecuencia vale la pena mencionar:

1. Elaboración incompleta de la información con omisión de variables importantes que facilitarían posteriormente su análisis.

2. Error de diagnóstico al ingreso.

3. No se definen pautas de manejo y conducta a seguir, con base en una sospecha clínica inicial.

4. En ocasiones no existe una epicrisis o cierre completo de la historia.

La situación planteada con las historias incompletas se subsanó en gran parte con el análisis al fondo hecho por los autores del trabajo, quienes hicieron una revisión exahustiva de toda la documentación existente en cada caso a partir del archivo de Anatomia Patológica, Laboratorio Clínico, Libros de Registro existen tes en admisión, Salas de Parto, Cirugía, Servicio de Sépticas y Servicios de Toxemias entre otros. De esta manera se obtuvo una información más completa que permitió llegar a un juicio objetivo sobre cada muerte ocurrida en la Institución.

\section{A - TASA DE MORTALIDAD}

En los dos años revisados en el I.M.I. (1981-1982), se presentaron 66 muertes maternas que cumplían ampliamente la definición de la FIGO para ser consideradas como tales. Durante el mismo tiempo hubo en la Institución veinte mil quinientos veinticuatro (20.524) nacimientos de niños vivos. Al relacionar estas dos cifras se obtuvo una tasa de mortalidad de $32.64 \times 10.000$ nacidos vivos.

La tasa anterior modifica la tendencia descendente de la mortalidad materna en el I.M.I. en los últimos 20 años $(10,10$, 17). Como se observa en el Cuadro siguiente:

Tabla No. 20

TASA DE MORTALIDAD

\begin{tabular}{lll}
\hline Autor & Años & Tasa \\
\hline López Ruíz & $1960-65$ & $45.4 \times 10.000$ \\
Rojas y Colaboradores & $1966-70$ & 35 \\
Sánchez F. & $1971-75$ & 30.4 \\
Muñoz y Colaboradores & $1976-80$ & 25.04 \\
Muñoz Becerra & $1981-82$ & 32.64 \\
\hline
\end{tabular}

Al comparar la tasa anterior con la mostrada en estudios realizados en otras Instituciones Universitarias y Hospitales Generales del país se aprecia cómo nues- 
tra Institución tiene uno de los índices más altos de mortalidad $(3,7,19)$. Ver Tabla 21.

Tabla No. 21

\begin{tabular}{lll}
\hline & Años & Tasa \\
\hline Valle (Cali) & $1977-78$ & 42.9 \\
I.M.I. (Bogotá) & $1981-82$ & 32.64 \\
$\begin{array}{l}\text { Hospital San Vicente } \\
\text { de Paul (Medellín) }\end{array}$ & $1968-72$ & 27 \\
$\begin{array}{l}\text { Estudios hospitalarios } \\
\text { de mortalidad materna }\end{array}$ & $1979-80$ & 23.1 \\
\hline
\end{tabular}

Resulta mayor aún la cifra de mortalidad encontrada en esta revisión si la cotejamos con la notificada por Centros Hospitalarios del área Latinoamericana comparables por muchos aspectos con el nuestro $(1,2,4,9,14)$.

Tabla No. 22

\begin{tabular}{lllll}
\hline Hospital & Ciudad & & Af̆́os & Tasa \\
\hline I.M.S.S. & México & & 1976 & 10.75 \\
Hospital Universitario & Maracaibo & & $196.1-70$ & 14.20 \\
Hospital "20 de Noviembre" & México & & $1976-79$ & 14.60 \\
Hospital "Concepción Palacios" & Caracas & & $1939-74$ & 15.9 \\
I.M.I. & Bogotá & & $1981-82$ & 32.64 \\
\hline
\end{tabular}

La tasa encontrada en el I.M.I. aparece extremadamente elevada al relacionarla con Instituciones Hospitalarias de países desarrollados en donde practicamente las causas determinantes de esta mortalidad están controladas $(2,5,14)$. Ver Tabla 23.

Tabla No. 23

\begin{tabular}{llll}
\hline Hospital & Ciudad & Años & Tasa \\
\hline Pensilvania & Pensilvania & $1959-73$ & $3.4 \times 10.000$ \\
Hutzel & Detroit & $1976-80$ & 2.4 \\
Milwakee & Wisconsin & 1977 & 1.86 \\
Landesfranenklinik & Wuppeutel & $1973-79$ & 1.6 \\
\hline
\end{tabular}

Más adelante analizamos los factores que pueden estar incidiendo en la tasa de mortalidad del I.M.I. y trataremos de demostrar como sería conveniente establecer una tasa depurada que reflejara de manera más directa la calidad de la atención obstétrica dispensada por un organismo de salud determinado.

\section{B - CAUSAS DE MORTALIDAD MATERNA}

El número mayor de muertes maternas se encontró en el grupo de causas directas con un total de (58 casos) equivalente a $87.9 \%$ sobre la mortalidad global. Sigue en este orden las causas indirectas 6 casos, $9.1 \%$ y finalmente, las causas de orden no obstétrico 2 casos, $3.1 \%$.

\section{C - MORTALIDAD MATERNA DE CAUSA DIRECTA}

Dentro de este grupo la infección ocupa el primer lugar ( $86.2 \%$ ) seguida de lejos por la toxemia (6.9\%) y la hemorragia $(5.2 \%)$, solamente se registró un caso de embolia de líquido amniótico.

Este ordenamiento de las causas directas de mortalidad es igual al encontrado en los últimos 20 años en el Instituto Materno Infantil I.M.I. y otras Instituciones $(1,3,4,7,10,16,17)$.

\section{Sepsis obstétrica}

Resalta en relación con el trabajo anterior de mortalidad materna, del I. N.I. (1976-1980), como la infección muestra un ascenso vertiginoso al pasar de un porcentaje de $69.7 \%$ a $86.2 \%$. La sepsis obstétrica se sigue presentando como consecuencia del aborto provocado (68\%) y por complicaciones del puerperio después de parto o cesárea $(30 \%)$.

Al revisar las muertes ocurridas en el puerperio a causa de la infección (15 casos), se estableció claramente como el 
$60 \%$ de las pacientes fueron remitidas al I.M.I. en estados sépticos avanzados después de parto o cesárea practicados en Hospitales de atención intermedia de Bogotá $y$ de poblaciones vecinas. El $40 \%$ restante presentaron infecciones después de parto 3 casos, y cesárea 3 casos, en el I.M.I.

Las cifras anteriores demuestran claramente como el manejo y la remisión de pacientes sépticas no se hace en forma oportuna al nivel correspondiente. Lo anterior unido al grado de desnutrición, anemia, malas condiciones socio-económicas $y$ ambientales que tienen estas enfermas hace que la sepsis tenga una evolución rápida y nefasta en la mayoría de ellas.

\section{Toxemia}

En los últimos estudios de mortalidad materna realizados en el I.M.I. se viene notando un descenso franco en el grupo de pacientes muertas por toxemia (10, $15,16,17)$. Esta baja de la mortalidad resalta aún más en el lapso revisado (1981-1982) en donde el porcentaje por esta causa se redujo a la mitad.

Los factores que han contribuido a mejorar la evolución de las pacientes toxémicas en el I.M.I. están dados en parte el mejor conocimiento de la fisiopatología de la entidad lo cual ha permitido cambios substanciales en el tratamiento médico y conductas obstétricas más agresivas.

De los tres casos encontrados de hemorragia, uno de ellos llegó al I.M.I. con cuadro de shock anémico irreversible por placenta previa a quien no se pudo dar tratamiento oportuno por tener un grupo sanguíneo de difícil consecución.

Los dos casos restantes no presentaron complicaciones atribuibles directa- mente a la hemorragia como causa primaria sino que ella apareció como complicación secundaria de un proceso de C.I.D. desencadenada por un abruptio placenta y una enfermedad trofoblástica.

La baja mortalidad materna atribuible a la hemorragia en el I.M.I. se debe en buena parte a un mejor manejo de la conducción y atención del parto, conductas más activas en el manejo de las causas de hemorragia en el tercer trimestre por parte del personal médico.

Otro hecho importante ha sido el establecimiento de un verdadero banco de sangre con una dotación adecuada y servicio permanente que permite tratar en forma oportuna las urgencias derivadas de esta causa.

A pesar de los esfuerzos realizados en el I.M.I., para mejorar el manejo de la toxemia, aún siguen Ilegando pacientes con embarazo a término y complicaciones irreversibles producidas por la entidad en referencia. La situación anterior es fácil de corregir, si se consigue mejorar la atención prenatal a nivel de los diferentes organismos de salud de Bogotá. En esta forma se lograría detec. tar precozmente los signos y síntomas que obligarían a una pronta remisión de estas enfermas al nivel correspondiente; allí mediante hospitalización y atención oportuna es posible prevenir las secuelas irreparables de la enfermedad.

\section{Hemorragia}

La hemorragia como causa de muerte en el I.M.I. ha disminuido en los últimos años. Vemos como el porcentaje inicial que era cerca del $20 \%$ ha llegado a $5.2 \%$ cifra encontrada en el presente estudio.

Las causas determinantes de la hemorragia también han variado a través del tiempo; así vemos como la atonía uterina, ruptura uterina, acretismo placen- 
tario, placenta previa y abruptio placenta, reportada por otros autor-s con alta incidencia, no se encontró en la revisión actual.

\section{D - MORTALIDAD DE CAUSA INDIRECTA}

Dentro de este grupo la enfermedad vascular crónica hipertensiva (E.V.C.H.) está en primer lugar con $66.6 \%$ Al compararla con las causas de mortalidad global comparte el segundo puesto junto con la toxemia.

Las cifras anteriores no muestran cambio favorable en relación con el trabajo realizado en el período (19761980), antes por el contrario indican estancamiento de esta entidad.

El análisis conjunto de las causas indirectas de muerte materna en el presente estudio: (Enfermedad vascular crónica hipertensiva, Cáncer de seno y diabetes) nos permite deducir que estas muertes podrían ser evitables en alto porcentaje, si a estas mujeres se les hubiera dispensado una asesoría médica oportuna, con el fin de disuadirlas de un nuevo embarazo de manera temporal o definitiva.

\section{E - MUERTE NO MATERNA}

Dentro de las causas consideradas como no obstétricas en la mortalidad materna, se encontraron dos complicaciones surgidas ambas en el puerperio; una a partir de una hepatitis y otra de una apendicitis; en ambos casos el diagnóstico fue tardío y las pacientes ingresaron al I.M.I. en estado terminal. Esta situación que ha sido encontrada en otros trabajos de mortalidad, pone de presente como se olvida por parte del médico que la mujer en estado grávido puerperal puede presentar cualquier patología médico-quirúrgica, que si bien se enmascara por el embarazo obliga al clínico a agudi- zar sus sentidos con el ánimo de hacer un diagnóstico oportuno y ofrecer un tratamiento adecuado que evite complicaciones funestas.

\section{F - CAUSA DE MORTALIDAD MATERNA CON RELACION A: EDAD, NUMERO DE GESTACIO- NES Y ESTADIO DEL EMBARAZO}

La mayor mortalidad en relación con edad materna, se presento en el grupo de mujeres de 20 a 29 años a causa de la infección derivada del aborto provocado $(59.1 \%)$. Sigue en orden el grupo de mujeres mayores de 35 años (21.2\%) en donde las entidades responsables del fallecimiento fueron: la infección, hemorragia y la E.V.C., en proporciones similares.

Al relacionar las variables número de gestaciones y causa de muerte, se aprecia como las grandes multigestantes, junto con aquellas que cursaban su primer embarazo, tienen los índices más altos (51.5 y $19.6 \%$ ) respectivamente. La causa de muerte en ambos grupos tuvo un común denominador en la sepsis obstétrica a expensas principalmente del aborto provocado.

Los estadios de la gestación en donde ocurrió mayor mortalidad fueron primer trimestre del embarazo (50\%), puerperio (33\%) y tercer trimestre (12\%). En el primer grupo la causa de muerte fue la infección post-aborto provocado. En el segundo grupo la infección puerpera! adquirida después del parto o cesárea; y en el último grupo la causa primordial estuvo en el A.C.V. como consecuencia de la E.V.C.H.

Es cuando menos preocupante de acuerdo con las cifras obtenidas al correlacionar las variables causa de muerte con edad materna, número de gestaciones $y$ estadio del embarazo, ver que el aborto provocado es utilizado como "método de planificación" por mujeres 
en cualquier edad de la vida reproductiva $y$ especialmente por multigestante.

Como consecuencia de la práctica del aborto provocado, realizado por personal empírico y en condiciones carentes de normas mínimas de asepsia y técnicas adecuadas, nuestras mujeres de estratos socioeconómicos bajos, están pagando no sólo con su vida la interrupción del embarazo, sino que además destruyen seriamente el núcleo familiar, principio de toda sociedad en donde la madre es el pilar más importante, dejando en la orfandad a sus hijos quienes más tarde acrecentarán los problemas sociales de nuestro país.

Llama la atención el elevado número de mujeres que mueren en el puerperio a consecuencia de cuadros sépticos derivados de un mal manejo en la atención del parto o cesárea. Ambas situaciones atribuibles en parte a las Instituciones Hospitalarias y a su cuerpo médico que interviene en el tratamiento de estas pacientes.

Igual comentario merecen las muertes ocurridas en el tercer trimestre a consecuencia de la E.V.C.H., que como se dijo en la parte relacionada con esta entidad, son inuertes evitables; con una consejería adecuada a estas mujeres, antes de pensar en un nuevo embar azo, sería posible disuadirlas en su deseo cuando exista contraindicación médica absoluta y en otros casos de menor riesgo se les motivaría a seguir un control prenatal riguroso.

\section{G - ANATOMIA PATOLOGICA}

La práctica de la autopsia se realizó en 18 pacientes (27\%). Los hallazgos quirúrgicos encontrados durante intervenciones practicadas días antes de la muerte, a un grupo de 37 pacientes (56\%) y su correspondiente estudio histológico de especímenes (extirpados), permitió una amplia confirmación clínico patológica del diagnóstico. Este hecho fue tenido en cuenta por los patólogos quienes no justificaron practicar estudio post-morten en estos casos especialmente de sepsis, donde el cortejo de complicaciones se conoce ampliamente.

En once pacientes no se practicó autopsia (16\%) las causas fueron levantamiento médico legal 6 casos y oposición de los familiares 5 casos.

Los datos anteriores demuestran plenamente como el diagnóstico clínicopatológico estuvo respaldado en el presente estudio en un $83 \%$. Esta cifra es bastante buena para un estudio de mortalidad en nuestro medio y da un amplio respaldo a los resultados presentados.

\section{H - MORTALIDAD MATERNA EN RELACION CON REMISION DE PACIENTES}

Sobre el total de las 66 pacientes fallecidas, 33 consultaron directamente a la Institución; la revisión cuidadosa de las historias clínicas de este grupo de pacientes mostró lo siguiente: 22 pacientes (66.6\%) ingresaron con complicaciones severas a partir de aborto provocado, toxemia y hemorragia. Once (11) pacientes 33.3\% fueron admitidas para atención de raspado por aborto incompleto provocado (4), parto (3) y cesárea (3) y de E.V.C.H. más embarazo (1). Estas pacientes presentaron complicaciones posteriores atribuibles al manejo dispensado en la Institución.

El $50 \%$ restante de las mujeres muertes (33 casos) consultaron primero a organismos de salud de la Regional No. 3 y de otras regionales de Bogotá, hecho este que pone de presente una vez más como el I.M.I. es un Hospital de referencia de cualquier zona de la ciudad y poblaciones vecinas. 
Desafortunadamente en el Distrito Especial de Bogotá la regionalización en la atención de salud y la remisión de pacientes no opera en la forma establecida por el sistema nacional de salud y menos aún teniendo en cuenta la sectorización en que se encuentra dividida la ciudad, con base en los diferentes niveles de atención existentes.

De las pacientes remitidas el (9.1\%), procedía de Hospitales Universitarios del Distrito Especial de Bogotá, donde existen los recursos necesarios para la atención del alto riesgo obstétrico. En ocasiones no se presta el servicio a pacientes carentes de medios económicos suficientes y necesarios en la atención de su estado, así la enferma pertenezca al área de influencia.

En los casos restantes (24 pacientes) la remisión fue de hospitales de atención intermedia de Bogotá y pueblos vecinos, en donde la complicación de sepsis obstétrica se presentó después de parto o cesárea. El traslado de estas pacientes al I.M. I. se hizo siempre en forma tardía.

La falla anotada en la regionalización y remisión de pacientes al nivel de atención correspondiente hace que el I.M.I. soporte una carga asistencial mayor a sus propias capacidades y recursos disponibles. Además, el recibir pacientes remitidas con complicaciones irreversibles eleva su tasa de mortalidad sin que ello refleje de manera objetiva la calidad de la atención ofrecida por la Institución.

\section{I - ATENCION INSTITUCIONAL Y MEDICA}

No es fácil determinar con precisión la existencia de responsabilidades del personal médico o paramédico que tuvo a cargo la atención de una enferma por parte de una Institución.
Sabemos de antemano cómo existe una serie de factores entrelazados cuyo conjunto contribuye de una manera o de otra en su resultado final.

Más difícil se hace aún establecer o no existencia de falla institucional o médica en relación con la muerte de una paciente, especialmente cuando se trata de mujeres en quienes factores culturales, sociales, económicos y religiosos inciden de manera desfavorable en la progresión de su enfermedad.

Dejando de lado las consideraciones anteriores es útil para el hospital y su departamento respectivo establecer mediante este indicador de atención institucional y médica una auditoría permanente, cuyo objetivo principal debe ser el despertar en las directivas administrativas, académicas, personal médico y paramédico el sentido de responsabilidad que a cada uno de ellos compete.

Se demostró a través del juicio crítico hecho a cada historia clínica analizada, cómo la falta de recursos institucionales necesarios en la atención o las condiciones derivadas de la planta física actual del hospital contribuyó en un $10.7 \%$ de manera desfavorable en la evolución posterior de las pacientes.

De igual manera se encontró en relación con la atención médica que el diagnóstico equivocado al ingreso en algunos casos, seguimiento inadecuado en otros, o la demora en la aplicación del tratamiento médico o quirúrgico, favoreció el empeoramiento del estado general de las pacientes en un $13.7 \%$.

En relación con otros trabajos de mortalidad realizados en el I.M.I. y otras instituciones, las fallas institucionales muestran tendencia a aumentar. En cambio el error médico ha disminuido en forma significativa, sin tener cifras que se puedan considerar ideales en un hospital universitario $(7,16,17)$. 


\section{II - TASA DEPURADA DE MORTALIDAD MATERNA EN EL I.M.I.}

Creimos importante tratar de establecer el número de pacientes, cuya causa de muerte sea atribuible directamente al hospital en razón al manejo y cuidados dispensados dentro de la institución. De acuerdo con el comentario hecho anteriormente en relación con las pacientes remitidas al I.M.I. así como los que, ingresaron directamente a él, se estableció que en once pacientes (16.6\%) sobre el gran total de las 66 fallecidas, la complicación obstétrica que las llevó a la muerte fue iatrogénica. Al correlacionar el total de muertes atribuibles al I.M.I. con el número de nacidos vivos en los dos años analizados (20.524) tendríamos una tasa de mortalidad depurada de 5.36 por 10.000 nacidos vivos, cifra está que si refleja la calidad de la atención dispensada por el nivel universitario de la Regional No. 3.

\section{CONCLUSIONES}

1. La tasa de mortalidad materna en el I.M.I. de Bogotá durante los años 1981-1982 fue de 32.64 por 10.000 nacidos vivos.

2. Durante los dos años analizados 1981-1982 se observó un aumento en la tasa de mortalidad de la institución.

3. La mortalidad más alta se encontró, en el grupo de causa directa $87.9 \%$ seguidas de las causas indirectas $9.1 \%$ y las no obstétricas $3.9 \%$.

4. Entre las causas directas la infección ocupa el primer lugar 86.2\% seguida de la toxemia $6.9 \%$ y la hemorragia $5.2 \%$.

5. El aborto provocado es la principal causa de sepsis obstétrica (68\%).
6. Es alto el porcentaje de infección puerperal $(30 \%)$ a partir de parto o cesárea.

7. La tasa de mortalidad por toxemia continúa descendiendo en forma ostensible en el I.M.I.

8. La mortalidad materna de causa indirecta está dada principalmente, por E.V.C.H., que ocupa junto con la toxemia el segundo lugar dentro de las causas globales.

9. La mortalidad materna de causa indirecta, se considera evitable en la mayoría de los casos.

10. En los grupos de 20 a 29 años (59.1\%) y mayores de 35 años $(21.2 \%)$ se presentó la mayor mortalidad a causa del aborto provocado.

11. En pacientes multíparas (51.5\%) y primigestantes $(19.6 \%)$ se encontró el índice más alto de mortalidad a expensas del aborto provocado.

12. La muerte ocurrió con mayor frecuencia en el primer trimestre de gestación $50 \%$, puerperio $33 \%$ y tercer trimestre $12 \%$

13. El $50 \%$ de las pacientes fallecidas fueron remitidas al I.M.I. de otras instituciones con complicaciones severas establecidas.

14. La atención institucional y médica contribuyó desfavorablemente en el resultado final en un $10.7 \%$ y $13.7 \%$ respectivamente.

15. Se establece una tasa depurada de mortalidad materna en el I.M.I. de $\mathbf{5 . 3 6}$ por 10.000 nacidos vivos.

\section{RECOMENDACIONES}

El análisis de los resultados obtenidos en éste estudio muestra una vez más có- 
mo la mortalidad materna en nuestro país, entraña un problema de salud pública de gran magnitud, en donde a pesar de existir políticas de atención materno-infantil muy definidas por parte del Ministerio de Salud, Seccionales y Departamentos Universitarios de Obstetricia y Ginecología, estas no se reflejan en beneficios concretos sobre el grupo etario hacia el cual están dirigidas.

La afirmación anterior se demuestra fácilmente al ver cómo la tasa de mortalidad materna registrada en las ínstituciones como la nuestra se eleva en forma considerable y se mantienen sin cambios los factores que están incidiendo directamente en el fallecimiento de la mujer grávida.

Sería importante acabar con el paralelismo existente en la formulación y aplicación de políticas entre las diferentes entidades encargadas de hacerlo, buscando mecanismos que permitan integrar a través de la Sección MaternoInfantil del Ministerio de Salud el recurso humano necesario de los diferentes organismos destinados a la atención materna, quienes con base en políticas definidas y programación académica elaboren planes conjuntos para desarrollar a través del recurso físico y humano disponibles en cada regional. Esto redundará en una mejor asistencia y necesariamente una mejor docencia.

Una vez establecida la integración docente-asistencial será posible integrar y capacitar a todo el personal participante del equipo en los diferentes programas dirigidos a la comunidad, tales como edu cación en salud, planificación familiar, consejería en reproducción humana, control prenatal, atención obstétrica $y$ post-parto.

De esta forma se lograrán unificar los criterios y las conductas que se deben seguir con cada paciente frente a una entidad definida y de acuerto con el nivel de atención correspondiente según lo establezca la clasificación del riesgo.

En relación con el recurso institucional disponible en cada nivel de atención se hace necesario establecer servicios que permitan prestar una atención integral a la paciente obstétrica en hospitales de atención secundaria y terciaria. Entre estos servicios vale la pena mencionar el de sépticas y toxemias. Sólo así podemos evitar que pacientes vistas en instituciones diferentes al I.M.I. puedan ser manejadas en la mayoría de los casos en estos organismos en forma oportuna y adecuada evitando su remisión tardía cuando ya las secuelas establecidas son irreparables.

El planteamiento anterior presupone la puesta en marcha de mecanismos que garanticen la regionalización y sectorización de los diferentes niveles de atención a través de los cuales se preste un servicio adecuado y gradual a los usuarios que lo soliciten.

En relación con las causas determinantes de la mortalidad materna el aborto no sólo sigue siendo la primera de ellas sino además aumenta cada día en una progresión alarmante.

Sabemos que la solución del problema es muy compleja por existir dentro de él factores de diferente índole que no están a la mano del médico para resolverlos. Aparte de la educación integral en salud que ya hemos mencionado que comprende necesariamente la educación sexual y planificación familiar, es importante que el Ministerio y los Servicios de Salud ejerzan una Auditoría Permanente sobre el ejercicio ilegal de la profesión, practicado por personal médico y empírico quienes son responsables de las complicaciones derivadas del aborto.

A través de una motivación directa hecha en pacientes detectadas como de 
altísimo riesgo para un nuevo embarazo es posible lograr la aceptación de evitarlo en forma temporal o permanente $y$ de esta manera se reducirán las muertes de causa indirecta.

Finalmente, es necesario recomendar en el I.M.I. la aceptación oficial del formulario elaborado en la recolección de

\section{BIBLIOGRAFIA}

1. AQUERO y Cols. Mortalidad materna en el hospital Conceprión Palacios de Caracas (1964-1972). Obstetricia y Ginecología Mexicana 35-590. 1974.

2. ALVAREZ BRAVO. Mortalidad materna en el mundo actual. Gaceta Médica Mexicana. 104. 25-30, 1972.

3. DIAZ LLANOS. Mortalidad materna en el Hospital Universitario del Valle. Acta Médica del Valle. Volumen 10 No. 3, 4. $112,118.1979$.

4. GARCIA y Cols. Mortalidad materna en el Hospital Provincial de Maternidad de Buenos Aires. Obstetricia y Ginecología Latinoamericana. 29: 478, 1971.

5. GUHA RAY. Maternal Mortality in Urban Hospital Maggicc Women's Hospital. Obstetric and Ginecology 17: 430, 1976.

6. International Federation of Ginecology and Obstetric. Report on the activity of the Comitte on the Maternal Mortality. Journal International Federation Obstetric and Ginecology. 5: 302, 1967.

7. JUBIZ y Cols. Muertes maternas en el H.S.V.P. Revista Colombiana de Obstetricia y Ginecología. Volumen XXIX No. 4. 178-82. 1978.

8. JUVIZ A. y SANCHEZ C., Mortalidad materna comparativa entre dos períodos 1963-67, 1968-72. Revista Colombiana los datos del presente estudio con el fin de ser diligenciado por parte del médico que cierra la historia una vez ocurra la muerte de una enferma $y$ su envío inmediato al departamento con destino al Comité de mortalidad. Este procedimiento facilitará los estudins que sobre mortalidad materna se hagan posteriormente en la Institución

de Obstetricia y Ginecología. Volumen XXXVII No. 3 129-37, 1976.

9. KARCHMER y Cols. Estudios de mortalidad materna en México. Consideraciones médico-sociales. Gaceta Médica Méxicana. 109: 63-85, 1975.

10. LOPEZ RUIZ. Morbilidad y mortalidad en el Instituto Materno-Infantil. Revista Colombiana de obstetricia v Ginecología. Volumen XIX No. 4 julio-agosto de 1968.

11. LOPEZ RUIZ. Morbilidad y mortalidad en el I.M.I. Revista Colombiana de Obstetricia y Ginecología. Volumen XVI No. 4 julio-agosto de 1965.

12. LOZADA y Cols. Mortalidad materna en Hospital de Arequipa. Revista Peruana. Obstetricia y Ginecología. 15: 69, 1969.

13. MARMOL J.G. History of the maternal, mortality comitee in the U.S.A. Obstetric and Gynecology 34: 123-69.

14. O'CONNORS TIMOTHY. Mortalidad Perinatal evitable. Urgencias Obstétricas. Salvat 1982.

15. PERALTA y Cols. Estudio de mortalidad en el I.M.I. Revista Colombiana de Obstetricia y Ginecología. Volumen IX; enero-febrero de 1968.

16. ROJAS Laura y Cols. Mortalidad materna en el I.M.I. de Bogotá. Revista Colombiana de Obstetricia y Ginecología. Volu- 
men XXV No. 2 128-49, marzo-abril de 1974.

17. SANCHEZ TORRES. Mortalidad Materna en el I.M.I. 1971-1975. Revista Colombiana de Obstetricia y Ginecología. Volumen XXXVIII 217-228, 1977.

18. SANCHEZ TORRES. Factores que intervienen en la calidad de la atención Gineco-
Obstétrica. en América Latina. Revista Colombiana de Obstetricia y Ginecología. Volumen XXXVIII No. 3 179. 1982.

19. URIZA y Cols. Estudio Hospitalario Mortalidad Materna. Revista Colombiana de Obstetricia y Ginecología. Volumen XXXIII No. 5 325-36, septiembre-octubre 1982. 